\title{
OBSERVATORIO
}

\section{BIBLIOTECAS UNIVERSITARIAS: CABALGANDO LA TECNOLOGÍA, SIGUIENDO AL USUARIO}

\author{
Lluís Anglada
}

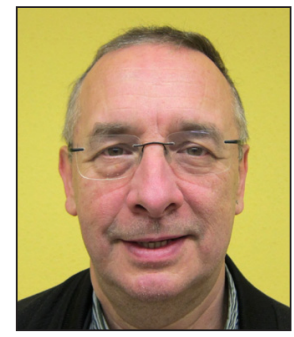

Lluís Anglada es director del Consorci de Biblioteques Universitàries de Catalunya (CBUC) desde 1997. Anteriormente fue director de las bibliotecas de la Universitat Politècnica de Catalunya y profesor de la Escola Universitària Jordi Rubió i Balaguer de Biblioteconomia i Documentació de Barcelona. Es licenciado en filosofía y diplomado en biblioteconomía y documentación. Ha sido miembro del Global Council de OCLC y actualmente pertenece a la Comisión Ejecutiva de Liber. Es autor y promotor de los blogs Bdig y Blok de Bid. Imparte regularmente talleres y conferencias y publica artículos sobre bibliotecas y consorcios bibliotecarios.

http://orcid.org/0000-0002-6384-4927

Consorci de Biblioteques Universitàries de Catalunya Gran Capità, 2-4, edif. Nexus, 4aㅡ pl., 08034 Barcelona, España langlada@cbuc.cat

\section{Resumen}

Repaso de los avances tecnológicos que más han influido en la evolución de las bibliotecas universitarias, y descripción de las actuaciones realizadas para adaptarse a los mismos: repositorios y acceso abierto, renovación de espacios, servicios a los investigadores, instrumentos de descubrimiento, y libro electrónico. Se concluye que en general las bibliotecas académicas gozan de buena salud gracias a que han sabido anticiparse a los cambios, y tienen buenas perspectivas de futuro.

\section{Palabras clave}

Bibliotecas universitarias, Tecnologías, Servicios, Adaptación, Cambio, Acceso abierto, Repositorios, Apoyo a la investigación, Documentos electrónicos, Herramientas de descubrimiento.

\section{Title: University libraries: riding the tecnology, keeping an eye on the user}

\section{Abstract}

Review of the technological advances that have influenced the evolution of the academic library, and description of the actions taken to accommodate them: developing repositories and open access, renovating spaces, providing services to researchers, offering search tools and ebooks. The general conclusions are that academic libraries are healthy because they have been able to anticipate change and that they have good prospects for the future.

\section{Keywords}

University libraries, Technologies, Services, Adaptation, Change, Open access, Repositories, Research support, Electronic documents, Ebooks, Discovery tools.

Anglada, Lluís. "Bibliotecas universitarias: cabalgando la tecnología, siguiendo al usuario". El profesional de la información, 2012, noviembre-diciembre, v. 21, n. 6, pp. 553-556.

http://dx.doi.org/10.3145/epi.2012.nov.01

\section{Introducción}

Podemos llamarlo tecnologías de la información o bibliotecas digitales (y hace unos años lo llamábamos automatización de bibliotecas), pero si miramos los impactos de la tecnología ${ }^{1}$ encontraremos muy pocas novedades (¿o ninguna?) que no se haya aplicado a los servicios prestados por las bibliotecas universitarias. Éstas llevan 40 años cabalgando la tecnología para conseguir continuar siendo útiles a sus usuarios. Las constantes innovaciones han ido acompañadas de llamadas a hacer cambios profundos en los roles de la biblioteca universitaria. Voces diversas ${ }^{2}$ han advertido que las transformaciones a las que nos conducían los cambios tecnológicos no eran ni cosméticas ni acumulativas, sino estructurales y disruptivas.

Los cambios a los que se han visto sometidas las bibliotecas universitarias no han sido menores. Un estudio reciente ${ }^{3}$ ha descrito un panorama apocalíptico en el que el incremento de costes, la aparición de alternativas viables a la biblioteca, 
el uso decreciente de los servicios y las nuevas necesidades de los usuarios forzaría a la biblioteca universitaria al cambio. Pero las bibliotecas universitarias han sido conscientes de la necesidad de adaptarse anticipadamente al entorno. Lo han hecho haciendo profundos cambios organizativos entre los que destaca la creación de consorcios, a finales de siglo y principios de éste, para mejorar su capacidad de compra, y lo hicieron antes de verse atenazadas por la disminución de recursos derivada de la crisis económica actual. Lo han hecho también haciendo evolucionar los roles tradicionales de la biblioteca hacia otros más adecuados con los tiempos, estando constantemente preocupadas sobre cómo mostrar a la sociedad que las sufraga los valores que aportan ${ }^{4}$.

Las bibliotecas universitarias, con mayor o menor consciencia de la profundidad del cambio, han estado en el pasado $-\mathrm{y}$ siguen estando en el presente- en la vanguardia de la aplicación de las novedades tecnológicas a sus servicios. Los principales terrenos en los que, en estos momentos, se esta innovando creo que son los cinco siguientes: repositorios y acceso abierto, renovación de espacios, servicios a los investigadores, instrumentos de descubrimiento, y libro electrónico.

\section{Repositorios y acceso abierto}

El movimiento del acceso abierto (OA) está celebrando su décimo aniversario y nadie duda que las bibliotecas han tenido un papel decisivo en los resultados que se han conseguido. El proceso de reforma de la comunicación científica no está de ninguna forma terminado ${ }^{5}$, ni aún predeterminado, pero parece que las universidades y centros de investigación no querrán ya prescindir del control sobre su producción científica. Poner ésta en abierto en alguna de las modalidades que tome el OA supone una inversión de tiempo que el investigador no siempre está dispuesto o preparado para realizar. En un pasado algo lejano, acceder a bases de datos remotas a través de la teledocumentación consumía un tiempo que los científicos podían emplear mejor si alguien -las bibliotecas- realizaba las búsquedas bibliográficas.

Actualmente, la implicación de las bibliotecas universitarias en llenar los repositorios de contenido permite que el inves-

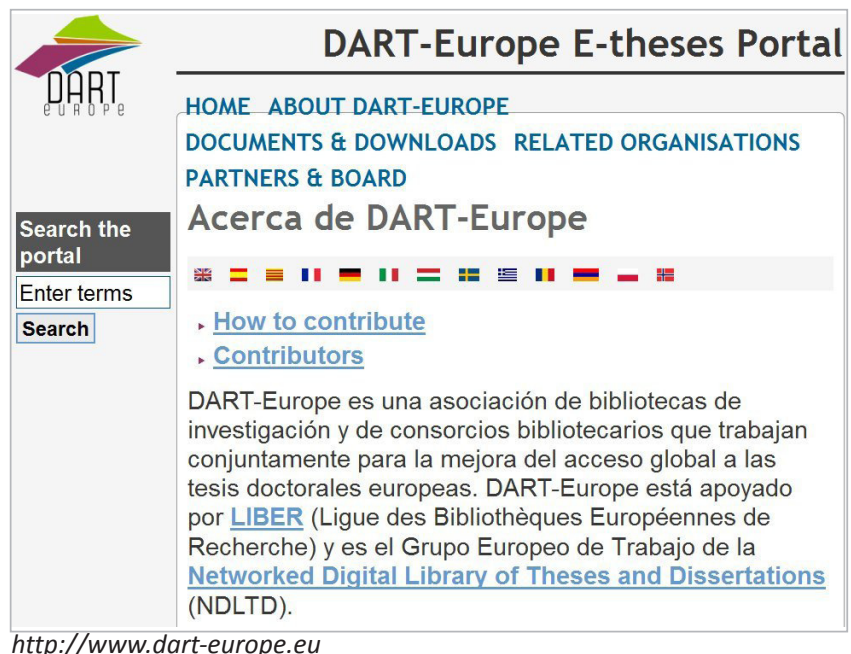

http://www.dart-europe.eu tigador ahorre un tiempo precioso y que la institución consiga mejor el objetivo final de tener archivada y accesible su producción científica. Probablemente el OA no vaya a tener una sola y simple forma, y a partir de esta diversidad las bibliotecas universitarias añadan a sus roles tradicionales el de asesorar a los autores sobre cuándo y en qué condiciones pueden publicar en abierto.

La progresiva digitalización de la vida universitaria llega a todos los rincones. Las tesis doctorales, uno de los productos más característicos de la universidad, quizá sean el ejemplo más claro de lo que puede suponer el movimiento del OA en un próximo futuro. Las tesis doctorales son recogidas a nivel institucional $y$, a través de protocolos de interoperabilidad, recopiladas por recolectores y puestas a disposición de usuario en portales supranacionales, como es el caso del proyecto DART Europe . $^{6}$

Las bibliotecas universitarias siempre han estado en la vanguardia de la aplicación de las novedades tecnológicas a sus servicios

\section{Espacios renovados}

A lo largo del siglo XX las bibliotecas universitarias crecieron ganando espacio para los documentos que conservaban, pero en la década de los 90 empezaron a renovar los espacios pensando ya más en los lectores que en los libros. Esta tendencia se ha visto enormemente reforzada por la progresiva utilización de revistas electrónicas, primero de forma complementaria a su versión impresa y más tarde de forma única. En poco más de quince años, grandes espacios de las dependencias de las bibliotecas universitarias ocupados por volúmenes encuadernados de revistas han dejado de tener sentido y han podido reorganizarse. Con respecto al uso de los espacios, los documentos han cedido protagonismo al usuario y las bibliotecas se han rediseñado y se están rediseñando para un uso más social de la información?. Las bibliotecas han creado nuevas áreas con salas para trabajar en grupo, se han dotado de muchos ordenadores y han buscado el confort de los usuarios introduciendo cafeterías. Algunos espacios nuevos han recibido nombres como learning commons, information commons o collaboratories y, en el mundo hispano, centros de recursos para el aprendizaje y la investigación (crais).

La digitalización de recursos y la renovación de los espacios ha traído una curiosa derivada: se necesitan espacios para almacenar los documentos impresos que las bibliotecas deben continuar conservando pero que tienen un uso bajo o nulo. Algunas bibliotecas ya habían creado almacenes de documentos fuera de su campus y en algunos casos estos almacenes eran cooperativos, pero en el horizonte de unos 20 años estas necesidades aumentarán. Ya no tiene sentido hacer almacenes propios cuando lo que se guarda en ellos puede consultarse digitalmente; por lo tanto, es lógico que las bibliotecas se planteen la conservación cooperativa de los documentos ${ }^{8}$ e incluso su complementariedad con los repositorios digitales de documentos ${ }^{9}$. 


\section{Servicios a los investigadores}

Los ordenadores conectados a las redes no sólo están cambiando los servicios bibliotecarios, cambian también la investigación. La bibliotecas universitarias tienen como una de sus principales misiones facilitar la investigación y lo han hecho tradicionalmente coleccionando documentos para los investigadores. Estos documentos hoy ya no son sólo libros o artículos de revistas, sino también datos. La eCiencia se desarrolla en redes y los distintos recursos que usan los científicos pasan a ser elementos de la infraestructura de la investigación ${ }^{10}$. En este nuevo entorno los retos para las bibliotecas son al menos dos: por un lado coleccionar y conservar un nuevo tipo de documentos (los conjuntos de datos), y, por otro, saber incorporarse a los procesos de la investigación para ser así útiles a los científicos.

Como en otros casos, la evolución tecnológica hace aparecer paradojas y la mirada de las bibliotecas se dirige a una parte de sus colecciones que tradicionalmente había estado 'escondida' y que ahora aparece como especialmente relevante para dar soporte a la investigación en ciencias humanas y sociales ${ }^{11}$.

\section{Instrumentos de descubrimiento}

En los años 80 del siglo pasado los opacs revolucionaron la forma de acceder a las colecciones de una biblioteca, pero ni los catálogos manuales ni los automatizados consiguieron nunca dar información sobre la totalidad de los documentos de que disponía una biblioteca. Las bases de datos complementaban el acceso para los artículos de revistas, pero esto requería hacer diversas consultas a sistemas que a menudo tenían interfaces distintas. Con los repositorios digitales, las bibliotecas universitarias se dotan aún de un tercer tipo de instrumento en el que conservan información. Demasiados receptáculos en un mundo en el que Google nos ha acostumbrado a obtener resultados con unas pocas palabras de búsqueda escritas en una única casilla.

\section{El investigador no siempre está dispues- to o preparado para realizar el depósito de sus trabajos}

Las herramientas de descubrimiento nacieron con la aspiración de ofrecer una experiencia de búsqueda distinta y enriquecida a los catálogos, y en este sentido, el primero que existió -AquaBrowser- se instaló a menudo como una 'piel' añadida al opac tradicional y no como substituto. Más o menos al mismo tiempo se desarrollaron buscadores federados -Metalib fue el primero de los comerciales- que permitían la consulta simultánea de varias bases de datos y ofrecían un solo conjunto de resultados. Pero el deseo (¿necesidad?) de los bibliotecarios de poder acceder a todos los recursos de la biblioteca desde un solo punto de consulta ha comportado que las búsquedas por filtraje y las federadas evolucionaran hasta convertirse en los productos que hoy se conocen como instrumentos de descubrimiento y que están aún en una fase no totalmente madura. Los catálogos se extienden para incluir búsquedas a artículos de revistas (como

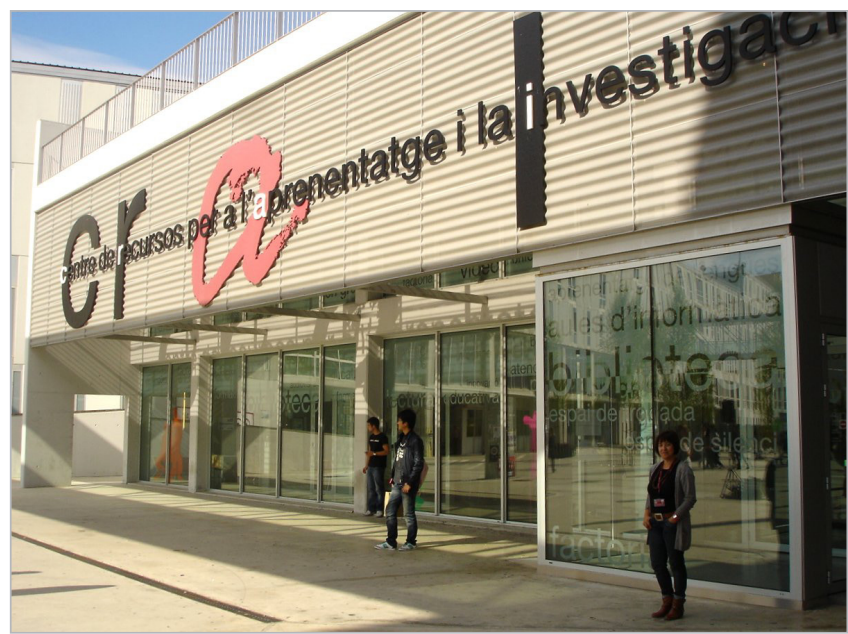

Uno de los crais de la Universitat Rovira i Virgili, Tarragona

hace actualmente WorldCat) y grandes índices de artículos de revistas pasan a incluir los catálogos de las bibliotecas y los contenidos de sus repositorios (como hacen Ebsco Discovery, Summon o Primo Central) ${ }^{12}$. Cualquiera de los dos caminos parece llevar al objetivo de permitir (iahora sí!) que una biblioteca ofrezca desde un solo punto de consulta el acceso a todos los recursos que posee o contrata (fondos impresos, documentos electrónicos contratados y objetos en repositorios digitales). Éste es en estos momentos uno de los desarrollos clave de las bibliotecas universitarias.

\section{Libros electrónicos}

Las bibliotecas universitarias han completado dos transiciones de lo impreso a lo digital: en primer lugar fueron los boletines de índices y resúmenes (revistas de abstracts) que pasaron al formato de base de datos hace unos 40 años. Más recientemente, las revistas científicas y académicas en texto completo. La transición hacia el libro electrónico empezó en los años 70 con el Proyecto Gutenberg pero sólo empezó a incidir y generalizarse en las bibliotecas hace unos diez años, cuando aparecieron los productos comerciales. A pesar de la experiencia previa de los editores y de las bibliotecas con los documentos electrónicos, los libros digitales no han explosionado como parecía que sucedería.

Seguramente los motivos son varios ${ }^{13}$, pero me parece que hay tres que son fundamentales:

- los editores no han sabido recrear el enorme fenómeno de succión que fueron los big deals para las compras consorciadas de paquetes de revistas;

- el mercado está ofreciendo los libros electrónicos desde diferentes plataformas y bajo diferentes modalidades de consulta y esta complejidad seguramente está inhibiendo muchos usos; finalmente,

- los editores, temerosos de la pérdida de mercado que les puede suponer el paso a lo digital, están entrando en este mundo poniendo bastantes restricciones a las bibliotecas en el uso de libros electrónicos.

Pero la extensión del uso de los ebooks es cuestión de tiempo. Mientras tanto se ha dejado sentir ya su influencia en la forma en que las bibliotecas compran. Antes adquirían libros en previsión de usos futuros (just-in-case), pero ahora 
algunas editoriales ofrecen compras en modalidad PDA ( $p a-$ tron driven acquisitions), es decir, poder comprar en función de las demandas de los usuarios. EI PDA consiste en que las bibliotecas puedan poner a disposición de sus usuarios fondos editoriales de libros digitales durante un plazo determinado de tiempo de manera que el uso de los libros proporcione datos que permitan a los departamentos de compras tomar decisiones en función de la demanda.

Al mismo tiempo, en el nuevo entorno digital, la edición universitaria tradicional ha entrado en crisis. La solución puede ser que las monografías universitarias se publiquen en electrónico en portales de agregadores, pero es posible también que se desarrollen nuevos modelos de negocio alrededor del acceso abierto. El futuro definitivo del libro electrónico no está aún escrito.

Por fin es posible que una biblioteca ofrezca acceso a todos los recursos que posee o contrata desde un sólo punto de consulta

\section{Conclusión}

Veo muy vivas las bibliotecas universitarias. En los últimos años han actuado proactivamente aprovechando las oportunidades que el entorno les ha presentado. Ni las compras consorciadas de recursos electrónicos, ni los repositorios institucionales ni el acceso abierto eran a priori terrenos destinados a que las bibliotecas se desarrollaran, y, sin embargo, han sido espacios de servicio que las bibliotecas universitarias han ocupado o ayudado a implementar. En plena efervescencia de las disrupciones que tienen lugar el mundo digital, las bibliotecas universitarias han sabido hacer evolucionar los servicios tradicionales a la vez que, cabalgando las tecnologías, han creado nuevos servicios siguiendo la estela de las necesidades en parte tradicionales, en parte cambiantes, de sus usuarios.

\section{Notas}

1. Véase el último número de la revista Library hi tech (2012, v. 30, n. 4) dedicado a '30 years of information technologies', y especialmente el artículo de Beth-Sandore Namachchivaya, "The first 30 years of the internet through the lens of an academic library: The University of Illinois at Urbana-Champaign, 1982-2012", pp. 623-642.

http://dx.doi.org/10.1108/07378831211285095

\section{Por ejemplo:}

Stoffle, Carla J.; Renaud, Robert; Veldof, Jerilyn R. "Choosing our futures". College \& research libraries, 1996, v. 57, n. 3, pp. 213-225.

Ross, Lyman; Sennyey, Pongracz. "The library is dead, long live the library! The practice of academic librarianship and the digital revolution". The journal of academic librarianship, 2008, v. 34, n. 2, pp. 145-152.

http://dx.doi.org/10.1016/j.acalib.2007.12.006

Coffman, Steve. "The decline and fall of the library empire". Searcher, 2012, v. 20, n. 12, p. 3.

http://www.infotoday.com/searcher/apr12/Coffman--TheDecline-and-Fall-of-the-Library-Empire.shtml

3. University Leadership Council. Redefining the academic library. Managing the migration to digital information services, The Advisory Board Company. Washington, DC, 2011. http://anatomiteca.com/2012/10/redefining-theacademic-library-managing-the-migration-to-digitalinformation-services

4. Ver la obra clave de Megan Oakleaf "The value of academic libraries", Association of College \& Research Libraries, 2010.

http://www.ala.org/acrl/sites/ala.org.acrl/files/content/ issues/value/val_report.pdf

5. Ver, por ejemplo, el debate suscitado por la publicación reciente (2012) del informe del grupo de trabajo del Reino Unido Expanding Access to Published Research Findings, liderado por Janet Finch: "Accessibility, sustainability, excellence: how to expand access to research publications". http://www.researchinfonet.org/wp-content/ uploads/2012/06/Finch-Group-report-FINAL-VERSION.pdf

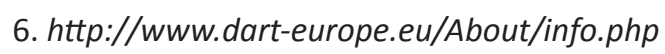

7. Appleton, Leo; Stevenson, Valerie. "Developing learning landscapes: academic library driving organizational change. Reference services review, 2011, v. 39, n. 3, pp. 343-361.

8. Payne, Lizanne. Library storage facilities and the future of print collections in North America. Dublin, Ohio: OCLC, 2007.

9. Malpas, Constance. Cloud-sourcing research collections: managing print in the mass-digitized library environment. Dublin, Ohio: OCLC, 2011.

10. Lossau, Norbert. "An overview of research infrastructures in Europe - and recommendations to Liber". Liber quarterly, 2012, v. 21, n. 3/4.

http://liber.library.uu.nl/index.php/lq/article/ view/8028/8386

11. Ver, por ejemplo el estudio de Dooley, Jackie M., and Luce, Katherine: Taking our pulse: The OCLC Research survey of special collections and archives. Dublin, Ohio: OCLC Research, 2010.

http://www.oc/c.org/research/publications/ library/2010/2010-11.pdf

12. Vaughan, Jason. "Web scale discovery services". Library technology reports, v. 47, n. 1. Chicago: ALA, 2011, 61 pp. ISBN: 9780838958292

13. Mcllroy, Thad. "Ebook formats are a mess - here's why". Learned publishing, 2012, v. 25, n. 4, pp. 247-250.

http://dx.doi.org/10.1087/20120402 